\title{
Advanced Practice Registered Nurse Onboarding and Orientation Program
}

\author{
Cheryl Lambert \\ Chamberlain University College of Nursing \\ Corresponding author : cherylalam@aol.com
}

\begin{abstract}
Background: The influx of advanced practice nurses into the healthcare industry has established the need for a postgraduate program for APRNs. Numerous studies have shown the efficacy of an onboarding process; however, there were limitations to the reviews.
\end{abstract}

Purpose: This study aimed to determine formal orientation and orientation programs, compared to current APRN orientation and orientation can improve the competence and quality of APRN care for eight weeks.

Methods: The participants were six currently practicing APRNs with eleven months to five years practicing as independent providers. The response rate in the pre-assessment phase was $100 \%$ and $66.7 \%$ in the post-assessment phase. Two APRNs came onboard after the start of the project, and one APRN opted out. There were no preceptors available for the completion of the CoEPCE competency tool. The APRN onboarding and orientation program utilized a competency tool with pre- and post-assessments. Emails with attached competency tool sent to APRNs for completion of pre- and post-assessments. The APRNs completed the pre- and post-competency assessments on the first day of implementation and the last day of week eight. The collection of data from the pre- and post-competency tool commenced in week nine and imported into Microsoft Excel for analysis. The data collected from the CoEPCE tool, as highlighted in the implementation phase, has significant changes

Results: The outcomes revealed that the changes were not significantly statistical at the plevel of 0.05 in the seven domains because none of the p-values were less than 0.05. However, the results revealed that deficits in statistical significance should not deem that the onboarding process was not successful.

Conclusion: The results demonstrated evidence of the importance of an APRN postgraduate program in enhancing competencies. However, larger sample sizes are substantial to show statistically changes for reliability and validity.

Keywords: Advanced practice registered nurse onboarding, orientation, nurse practitioner, transition to practice, postgraduate programs, competency tools, and preceptors. 


\section{BACKGROUND}

Advanced practice registered nurses (APRNs) are medical and mental health providers who are accountable and responsible who display commitment to patients, families, communities, and are in high demand (American Association of Nurses Practitioners, 2019). The 2025 projected shortage of healthcare providers has intensified the need for APRNs in clinical practice settings. The increase in the number of medical and mental health issues has heightened the demand for APRNs in healthcare settings (Brown, Poope, Kaminetzky, Wipf, \& Woods, 2015). ACA of 2010 and IOM recommend the implementation of a structured postgraduate program for APRNs. The need exists for these professionals to care for patients who are seeking care for the first time with an increased access to health insurance (Hicks, Rico, \& Beauchesne, 2018). The facility does not have an APRN onboarding and orientation program and a postgraduate program that aligns with ACA and Institute of Medicine (IOM) recommendation is significant for advance practice nurses' transition to practice (Hart \& Bowen, 2016). Certainly, organizations must stay cognizant of APRNs role in healthcare and provide the necessary resources to optimize patient care.

The complexity of care that patients require demands the undivided attention of APRNs. The Mental Health Parity and Addiction Equity Act (MHPARA), increasing suicide rates, and chronic diseases require that APRNs have organizational support and are familiar with the culture and the goals to provide optimal care (Painter, 2017). The implementation of an APRN onboarding and orientation program is vital for the organization to provide a smooth transition to clinical practice and continue to promote collaborative efforts for growth within the organization (Martsolf, Nguyen, Freund, \& Poghosyan, 2017).

Despite the need for advanced practices nurses, there is still a deficient of a formal postgraduate programs. APRN onboarding and orientation require partnerships within the organization to enhance proficiencies, strengthen relationships, build confidence, increase morale, improve quality patient care, and decrease turnover costs (Marshall \& Broome, 2017). Implementing a structured transition to practice is critical for promoting quality initiatives, especially with the demand for APRNs, which grew out of the need for increasing access to care, the aging population, and optimizing health. With the organization's goals and mission at the forefront of care, interprofessional collaboration is vital; therefore, the onboarding and orientation program must incorporate expertise in collaborative care (Goldsberry, 2018). Enhancement of skills and expertise is significant for APRNs to grow, and the organization needs to promote a culture of accountability and shared thoughts as well as expert support (Sherman \& Cohn, 2019).

Interprofessional collaboration is vital to enhance self-confidence and modernize quality patient care as well as focus on critical tools to improve competencies. The transformation from a novice APRN to an independent provider can be intimidating, which can disrupt patient care as well as affect turnover rates (Kirkman, Wilkinson, \& Scahill, 2018) Organizations must exhibit responsibility and support and provide an effortless shift into clinical practice (Kirkman, Wilkinson, \& Scahill, 2018; Klein, Polin, \& Sutton, 2018).

The support of nurse executives, the education department, APRNs, and the human resources department would enhance collaboration to foster the opportunity for an official onboarding and orientation program for professional development (Englebright, McCurley, \& Borum, 2017). The VA promotes a NP postgraduate residency program at several facilities that has validated NP graduates' competencies within their scope of practice. Data from various studies demonstrate that an APRN postgraduate program is 


\section{Journal Of Nursing Practice}

http://thejnp.org

ISSN: 2614-3488 (print); 2614-3496 (online)

Vol.3 No.2. April 2020. Page.253-267

necessary to facilitate growth, enhance competencies, and promote confident providers (Englebright, McCurley, \& Borum, 2017; Rugen, Dolansky, Dulay, King, \& Harada, 2018; \& Rugen, Speroff, Zapatka, \& Brienza, 2016).

Bush and Lowery (2016) reported that a structured and evidence-based APRN onboarding and orientation program would provide resilience in developing outstanding clinicians to facilitate skilled care in their respective fields. A formal onboarding and orientation process is of the essence to prevent burnout, increase self-confidence, promote an increased knowledge of relevant tools, and enhance patient-care resources (Bush \& Lowery, 2016). With healthcare rules and regulations that promote quality care and safety, organizations must continue to champion a culture of quality and safety and cultivate ongoing staff education. An APRN onboarding and orientation process will be necessary for APRNs to acclimate to the organization's culture, to enhance essential proficiencies, and to administer care aligning with the organization's goals (Moon, 2018).

This paper proposed an implementing of an evidence-based onboarding and orientation program for APRNs, focusing on the transition process into practice, enhancement of APRNs' competencies, and improvement of patient outcomes at a large healthcare organization in Augusta, Georgia. The results reported the significance of a formal onboarding practice utilizing an evidence-based competency tool created by the Veterans Administration (VA) Center of Excellence in Primary Care Education (CoEPCE) competency tool. The VA nurse practitioner (NP) residency program utilizes the CoEPCE's competency tool for outcome measures, and use as a guide for implementing an APRN postgraduate program.

\section{OBJECTIVE}

- To enhance interprofessional relationships within the organization by encouraging and motivating buy-in for the APRN program;

- To improve efficiency and performance measures of care delivery utilizing the VA CoEPCE competency tool;

- To measure participants' competencies utilizing a pre-and-post self-evaluation competency tool;

- To compare APRNs' pre-and-post self-assessment scores on achieving competencies; and

- To establish a successful transition into clinical practice for novice APRNs utilizing a pre-and-post self-evaluation competency tool

\section{METHODS}

There are ethical guidelines in place for human subjects protection, and Harris, Rousse, Dearman \& Thomas, (2016) reported that a person would not participate in a quality improvement initiative or research without informed consent. The DNP project is a quality improvement initiative and adhered to and aligned with the hospital Institutional Review Board (IRB) approval and standards. Bonnel and Smith (2017) shared that the Department of Health, Education, and Welfare (HEW) regulate the law that protects human subjects' rights, and IRBs have a unique role in maintaining compliance (p. 206). The DNP project manager completed the necessary human protection collaborative institutional training initiative (CITI) for compliance with ethical guidelines.

The DNP project did not include patients because the focus was on onboarding of advanced practice nurses; however, only APRNs participated in the program. Participants 


\section{Journal Of Nursing Practice}

http://thejnp.org

ISSN: 2614-3488 (print); 2614-3496 (online)

Vol.3 No.2. April 2020. Page.253-267

had information about the newly formed APRN orientation program during the NEO. The providers made their decisions for participation in the program. Informed consent was not deemed necessary because the advanced practice nurses' onboarding process was a extension of NEO.

The DNP project manager submitted requirements and approval from the IRB team. The DNP project manager adhered to IRB guidelines and requirements. To ensure compliance with the IRB's standards and to maintain ethical guidelines, the DNP project manager ensured participants' understanding the continuation from NEO sustaining APRNs' autonomy, encouraging respect and dignity, and preventing harm (Harris et al., $\underline{2016}$.

The participants were six currently practicing APRNs with eleven months to five years practicing as independent providers. The response rate in the pre-assessment phase was $100 \%$ and $66.7 \%$ in the post-assessment phase. Two APRNs came onboard after the start of the project, and one APRN opted out. There were no preceptors available for the completion of the CoEPCE competency tool.

The APRN onboarding and orientation program utilized a competency tool with pre- and post-assessments. Emails with attached competency tool sent to APRNs for completion of pre- and post-assessments. The APRNs completed the pre- and postcompetency assessments on the first day of implementation and the last day of week eight. The collection of data from the pre- and post-competency tool commenced in week nine and imported into Microsoft Excel for analysis. The data collected from the CoEPCE tool, as highlighted in the implementation phase, has significant changes

Although the sample sizes for each of these domain scores were small, the use of statistical testing to evaluate whether there was a statistically significant change in the scores in each domain. These tests did not have very much power due to the small sample sizes. Any lack of statistical significance does not imply that the onboarding and orientation program was ineffective. The relevance of choosing the correct statistical test in determining whether the changes in the scores are normally distributed. However, because the sample sizes for each test were small, any tests or visualizations to do this would be ineffective in demonstrating an increase in proficiencies. So, we will assume that the distributions in changes in the scores are normal; therefore, the use of paired t-tests to make these comparisons. This utilization of the test is appropriate to share outcomes with approximate continuous data and the pairing of the domain scores for the pre- and postimplementation outcomes.

Table 3 includes the results of paired t-tests for four of the domain scores. The three missing domains have sample sizes that were too small for analysis. One APRN scored at both time points in the quality improvement and population management domain. The other two scores that were missing did not have any variability in the changes that took place, and data was unavailable to perform the required statistical test. The three missing domains have sample sizes that have turned out to be too small for analysis.

Table 3 includes three statistics for each test. The $t$ statistic is a standardized version of the difference between the pre- and post-implementation scores and not directly interpreted. The $\mathrm{df}$ (degrees of freedom) is a way to discuss the amount of information available to perform the test; here, the value of 3 is the number of APRNs included (4) minus 1. The use of the $t$ statistics and the df to calculate the p-value demonstrated the results. An example is the p-value of 0.370 for clinical competency in planning and managing care means that if there has been no real change in this domain following the implementation, there is a $37 \%$ chance that we would have seen changes as substantial as those in Table 2 


\section{Journal Of Nursing Practice}

http://thejnp.org

ISSN: 2614-3488 (print); 2614-3496 (online)

Vol.3 No.2. April 2020. Page.253-267

just due to random variability in evaluations alone. That is not considered very small, especially compared to the normal level of significance of 0.05 , and so we cannot conclude that there has been a true, underlying change in this area. None of the $p$ values in Table 3 are less than 0.05 , so overall, we conclude that there have not been any statistically significant changes at the 0.05 levels in any of the competency tool domain scores following the implementation of the nurse onboarding and orientation program.

As previously mentioned, however, small sample sizes result in difficulty in statistically changes that there were in the scores. The small number of APRNs who participated in the project had scores that tended to go up following the implementation of this process, although not statistically significant. The small amount of practical evidence seems to indicate that the process is successful in increasing the competencies of the providers. A more extensive study would be required to understand whether this increase is significant.

\section{RESULTS}

Results describe the major findings of the study. It should be clear, concise and can be reported on texts or graphics. Please provide some introduction for the information presented on tables or images.

The competency tool included a total of 69 individual items; each required an evaluation of competencies on a scale from 1 ("observes task only") to 5 ("able to supervise others"). The scoring of the seven domains, by averaging the scores of the items associated with each domain. Those domains are clinical competency in planning and managing care, leadership, interprofessional team collaboration, and patient-centered care, shared decision making, and sustained relationships, and quality improvement, and population management. Each domain score is also on a scale from 1 to 5, with values near 1 indicating minimal competency, and values near 5 indicating an excellent level of competency.

There were six participants involved in the project. All six advanced practice nurses completed the pre-implementation assessments and four completed postimplementation assessments. Table 1 includes summary statistics for each of the seven domains of the pre- and post-competency tool. These summary statistics are appropriate for variables that are numeric and approximately continuous. There are many possible values between the lowest score of 1 and the highest score of 5 .

There are seven statistics associated with each domain at each time point.

- The "N" is the sample size, represents the number of APRNs who completed an assessment and evaluated for at least $80 \%$ of the items associated with that domain. An example of the sample size is four APRNs with a pre-implementation leadership score. The evaluation of six APRNs; however, two APRNs did not have enough items evaluated under the leadership scale for the measure of the domain to be valid.

- "Missing" is the number of participants who did not have a score, a lack of evaluation, or many items were missing.

- The mean is the average value. This value can help us understand how the "typical" APRN responded. Lower values indicate low competency, and higher values indicate excellent competency. Five is the highest possible value, and one is the lowest, overall. APRNs were considered to be on the more competent side of the scale on all measures at both time points. We can also see that for all measures, the mean scores are higher at post-implementation than pre-implementation, but we 


\section{Journal Of Nursing Practice}

http://thejnp.org

ISSN: 2614-3488 (print); 2614-3496 (online)

Vol.3 No.2. April 2020. Page.253-267

must be careful about interpreting this as a "change" because the same providers did not necessarily have a score at both time points.

- The median is another way of understanding the "typical" value. The median is the number that would be in the middle of all scores that are lined up from smallest to largest, referred to as the 50th percentile. These numbers are not very different from the means in the case of these nurses.

- The standard deviation is a way of understanding how much the APRNs' scores vary from each other. For example, the standard deviation of 0.396 for the preimplementation Clinical Competency in Planning and Managing Care domain means that a "typical" APRNs' score would be within plus or minus 0.396 points of the average of 3.83. More significant standard deviations mean the advanced practice nurses' scores are more different from each other, and smaller standard deviations mean the providers' scores are more similar to one another.

- Finally, the minimum and maximum values are the lowest and highest scores across the evaluation of APRNs. One value that stands out is a minimum score of 1.86 for the pre-implementation leadership domain; this was quite a low score relative to the standardized scores in other domains. Several perfect scores (5) patient-centered care, shared decision-making, and sustained relationships achieved perfect scores following the implementation.

All participants did not return a completed competency tool; we may be interested in seeing these scores and summaries of the changes for providers who scored at both preand post-implementation. See Table 2. The subtracting of the pre-implementation score from the post-implementation total was used to calculate changes in competencies.

When comparing scores for only the advanced practice nurses who had scored at both periods, all mean and median scores increase. The results are an indication that the training improved their competencies. The possibility that the scores may have increased due to outside influences since this was not a controlled experiment. Also, note the following from table two:

- The Quality Improvement and Population Management domain only had one APRN with both a pre- and post-implementation evaluation.

- The Leadership and Sustained Relationships domains both only had two APRNs with both a pre- and post-implementation evaluation; the standard deviation for both of these changes is 0 , which indicates that the two providers improved their scores by the same amount (no variability in the changes).

In addition to reviewing these tables, it can be helpful to visualize the changes in the scores. Because we are focusing on changes in the scores, Figures $1-7$ are bar charts that show mean pre- and post-implementation domain scores for only the APRNs who had scored for evaluations at both time points (like Table 2). These bar charts each have two bars: one for pre-implementation and one for post-implementation. The heights of the bars indicate the average scores in that domain, as indicated by the left (vertical) axis. All 7 bar charts show increases in the scores from pre-implementation to post-implementation.

Each of the bar charts shows the same increase in domain scores, as seen in Table 2. The findings indicate that there needs to be a formal onboarding and orientation program for APRNs to facilitate a smooth transition to practice, enhance competencies, augment the quality of patient care, reduce turnover rates, and lessen the costs to organizations to replace staff. These findings support previous studies, which enhances the body of knowledge indicative of a structured APRN onboarding and orientation process. The utilization of Benner's theory and Kotter's Change Model can direct APRN onboarding and 


\section{Journal Of Nursing Practice}

http://thejnp.org

ISSN: 2614-3488 (print); 2614-3496 (online)

Vol.3 No.2. April 2020. Page.253-267

orientation programs. Benner's theory can identify APRNs' needs based on their level of expertise to ensure appropriate tools are in place. Novice advanced practice nurses' needs are different from other skill levels of APRNs. Kotter's Change Model can smooth the progress of stakeholders' awareness, participation, and adaptability.

\section{DISCUSSION}

The discussion should explore the significance of the results of the study. The following components should be covered in discussion: How do your results relate to the original question or objectives outlined in the background section (what)? Do you provide interpretation scientifically for each of your results or findings presented (why)? Are your results consistent with what other investigators have reported (what else)? Or are there any differences?

The APRNs engaged in the project from September 4, 2019 to November 9, 2019 were currently practicing independent providers without a formal postgraduate program and two newly employed APRN. The participants' years of clinical practice ranged from 10 months to seven years. The project's aims and objectives were to demonstrate enrichment in competencies among APRNs to boost their confidence levels and improve patient care. The use of the CoEPCE competency tool demonstrates a minimal score of one and a maximum score of five. The option of checking off not applicable (N/A) did not contribute towards the total tally and was considered missing.

The results validated enhancement in competencies among APRNs after the conclusion of the eight-week implementation program. A paired t-test was significant in data analysis because of the small sample size. Six APRNs completed the pre-competency assessment; however, four completed the post-competency assessment. The comparison of the pre- and post-competency assessments was most significant to the DNP project. The pre-competency assessment affirmed that some APRNs scored at the higher end of the CoEPCE tool and years of nursing practice or previous independent provider clinical roles could have influenced the results.

The lowest scores in the pre-implementation phase were in the leadership domain because only four providers had scores for each item. The mean score exhibited maximum scores in all domains in the post-implementation phase; however, since some items were missing, the difficulty of arriving at a change in competencies cannot be determined at this time. The median values did not reflect any change from the scores of the mean, while the standard deviation demonstrated the differences and similarities in variations among APRNs' total scores. The post-competency scores in patient-centered care, shared decision-making, and sustained relationship domains revealed a maximum score of five. The omission of the following domains because of missing data: leadership, sustained relationships, and quality improvement, and population management. There were also incomplete assessments in clinical competency in planning and managing care, interprofessional team collaboration, and patient centered-care, but the values were large enough for analysis.

\section{CONCLUSION}

The healthcare industry has an enormous number of APRNs practicing as independent providers. With the mandate for enhancing quality and safety among patients nationally and globally, organizations must have a structured APRN onboarding an orientation practice in place. The utilization of the VA's one-year NP CoEPCE competency tool in the DNP project was to demonstrate the need for an APRN onboarding 


\section{Journal Of Nursing Practice}

http://thejnp.org

ISSN: 2614-3488 (print); 2614-3496 (online)

Vol.3 No.2. April 2020. Page.253-267

and orientation program. The approval to use and to modify the CoEPCE competency tool to align with an eight-week APRN onboarding and orientation program by one of the leaders at the VA's central office

The competency tool offers a guide for self-assessment by APRNs. The selfassessment allows the identification of areas of improvement and professional strengths. The EPA conceptual framework represents daily activities and applying competencies into data, and the logic model discusses the causes and effects of the desired outcomes. The utilization of the EPA and the logic model in the development of the CoEPCE competency tool demonstrates an evidence-based initiative with high reliability and internal consistency with a Cronbach alpha of 0.85- 0.95 (Harada et al., 2018).

The results of the APRN onboarding and orientation program validate the need for such a program that aligns with organizations' missions and goals. The findings shed light that the support and collaboration of stakeholders and APRNs are at the heart of the sustainability of a formal onboarding program. The sustainability of the APRN onboarding and orientation program is dependent upon the stakeholders' recognition of the program's alignment with the organization's strategic goals. Having outcome measures in place to monitor the program for continued success. The dissemination of the results of the DNP project will demonstrate the need for a supportive environment with transparency and engagement of both APRNs and leaders. 


\section{Journal Of Nursing Practice}

http://thejnp.org

ISSN: 2614-3488 (print); 2614-3496 (online)

Vol.3 No.2. April 2020. Page.253-267

\section{REFERENCES}

American Association of Nurse Practitioners. (2019). All about NPs and AANP. Retrieved February 25, 2020. From https://www.aanp.org/about.

Bonnel, W., \& Smith, K.V. (2017). Proposal writing for clinical nursing and DNP projects $\left(2^{\text {nd }}\right.$ ed.). New York, NY: Springer Publishing Company.

Brown, K., Poppe, A., Kaminetzky, C., Wipf, J., \& Woods, N. F. (2015). Recommendations for nurse practitioner residency programs. Nurse Educator, 40(3), 148-151. https://doi.org/10.1097/NNE.0000000000000117

Bush, C. T., \& Lowery, B. (2016). Postgraduate nurse practitioner education: Impact on job satisfaction. The Journal for Nurse Practitioners, 12, 226-234. https://doi.org/10.1016/j.nurpra.2015.11.018.

Englebright, J., McCurley, J., Borum, C. (2017). The emerging role of APRNs in hospital nursing practice: Perspectives from a survey of chief nursing officers. Nurse Leader, 15(6), 387-391. https://doi.org/10.1016/j.mnl.2017.09.004.

Goldsberry, J. W. (2018). Advanced practice nurses leading the way: Interprofessional collaboration. Nurse Education Today, 65, 1-3. https://doi.org/10.1016/j.nedt.2018.02.024.

Harada, N. D., Traylor, L., Rugen, K.W., Bowen, J. L., Smith, C. S., Felker, B., ...Gilman, S. C. (2018). Interprofessional transformation of clinical education: The first six years of the Veterans Affairs centers of excellence in primary care education. Journal of Interprofessional $\quad$ Care. https://doi.org/10.1080/13561820.2018.1433642.

Harris, J.L., Roussel, L., Dearman, C., \& Thomas, P. L. (2016). Project planning and management: A guide for nurses and interprofessional teams ( $2^{\text {nd }}$ ed.). Burlington, MA: Jones \& Bartlett Learning.

Hart, A. M., \& Bowen, A. (2016). New nurse practitioners' perceptions of preparedness for and transition into practice. The Journal for Nurse Practitioners, 12(8), 545552. https://doi: https://doi.org/10.1016/j.nurpra.2016.04.018.

Hicks, K. E., Rico, J., \& Beauchesne, M. (2018). Core curriculum and competencies: A multisite analysis of postgraduate training programs for primary care nurse practitioners. Journal of Professional Nursing, 34(6), 454-462. https://doi.org/10.1016/j.profnurs.2017.12.012.

Kirkman, A., Wilkinson, J., \& Scahill, S. (2018). Thinking about health care differently: Nurse practitioners in primary health care as social entrepreneurs. Journal of Primary Health Care, 10(4), 331-337. https://doi.org/10.1002/nur.21768.

Klein, H. J., Polin, B., \& Leigh Sutton, K. (2015). Specific onboarding practices for the socialization of new employees. International Journal of Selection \& Assessment, 23(3), 263-283. https://doi.org/10.1111/ijsa.12113.

Marshall, E.S., \& Broome, M.E. (2017). Transformational leadership in nursing: From expert clinician to influential leader (2nd ed.). New York, NY: Springer Publishing Company.

Martsolf, G. R., Nguyen, P., Freund, D., \& Poghosyan, L. (2017).What we know about postgraduate nurse practitioner residency and fellowship programs. The Journal for Nurse Practitioners, 13, 482-487. https://doi.org/10.1016/i.nurpra.2017.05.013.

Moon, H. (2018). Unleashing apprenticeship: From onboarding to professional development. European Journal of Training and Development, 42(1), 110-124. https://doi.org/10.1108/EJTD-06-2017-0056 
Painter, S. G. (2017). Opiate crisis and healthcare reform in America: A review for nurses. Online Journal of Issues in Nursing, 22(2), 11. http://dx.doi.org/10.3912/OJIN.Vol22No02Man03

Rugen, K. W., Dolansky, M. A., Dulay, M., King, S., \& Harada, N. (2018). Evaluation of veterans' affairs primary care nurse practitioner residency: Achievement of $\begin{array}{llll}\text { competencies. } & \text { Nursing }\end{array}$ https://doi.org/10.1016/j.outlook.2017.06.004

Rugen, K. W., Speroff, E., Zapatka, S. A., \& Brienza, R. (2016). Feature article: Veterans affairs interprofessional nurse practitioner residency in primary care: A competency-based program. The Journal for Nurse Practitioners, 12, e267-e273. https://doi.org/10.1016/j.nurpra.2016.02.023

Sherman, R. O., \& Cohn, T. M. (2019). Promoting professional accountability and ownership: Nursing leaders set the tone for a culture of professional responsibility. Retrieved March 2, 2020. From https://www.americannursetoday.com/promotingprofessional-accountability-ownership/ 


\section{Journal Of Nursing Practice}

http://thejnp.org

ISSN: 2614-3488 (print); 2614-3496 (online)

Vol.3 No.2. April 2020. Page.253-267

Table 1. Summary Statistics of All Competency Tool Domain Scores, Pre- and Post-

Implementation

\begin{tabular}{lllllllll} 
Competency Tool Domain & Time & $\mathrm{N}$ & Missing & Mean & Median & Std. Dev & Minimum & Maximum \\
\hline Clinical Competency in & Pre & 6 & 0 & 3.83 & 3.88 & 0.396 & 3.25 & 4.25 \\
Planning and Managing Care & Post & 4 & 2 & 3.97 & 4.00 & 0.156 & 3.75 & 4.11 \\
\hline Leadership & Pre & 4 & 2 & 3.11 & 3.14 & 1.214 & 1.86 & 4.29 \\
& Post & 3 & 3 & 4.14 & 4.00 & 0.247 & 4.00 & 4.43 \\
\hline Interprofessional Team & Pre & 6 & 0 & 4.02 & 4.00 & 0.528 & 3.22 & 4.89 \\
Collaboration & Post & 4 & 2 & 4.19 & 4.00 & 0.389 & 4.00 & 4.78 \\
\hline Patient-Centered Care & Pre & 6 & 0 & 3.86 & 4.00 & 0.645 & 3.14 & 4.86 \\
& Post & 4 & 2 & 4.25 & 4.00 & 0.500 & 4.00 & 5.00 \\
\hline Shared Decision Making & Pre & 6 & 0 & 3.90 & 4.00 & 0.421 & 3.14 & 4.43 \\
& Post & 4 & 2 & 4.25 & 4.00 & 0.500 & 4.00 & 5.00 \\
\hline Sustained Relationships & Pre & 4 & 2 & 3.83 & 4.00 & 0.451 & 3.17 & 4.17 \\
& Post & 3 & 3 & 4.39 & 4.17 & 0.536 & 4.00 & 5.00 \\
\hline Quality Improvement and & Pre & 3 & 3 & 3.47 & 3.60 & 0.231 & 3.20 & 3.60 \\
Population Management & Post & 3 & 3 & 4.02 & 4.00 & 0.225 & 3.80 & 4.25
\end{tabular}

Table 2. Summary Statistics of Competency Tool Domain Scores for Participants with Preand Post-Implementation Scores

\begin{tabular}{|c|c|c|c|c|c|c|c|}
\hline Competency Tool Domain & Time & $\mathrm{N}$ & Mean & Median & Std. Dev & Minimum & Maximum \\
\hline \multirow{3}{*}{$\begin{array}{l}\text { Clinical Competency in } \\
\text { Planning and Managing Care }\end{array}$} & Pre & 4 & 3.70 & 3.64 & 0.422 & 3.25 & 4.25 \\
\hline & Post & & 3.97 & 4.00 & 0.156 & 3.75 & 4.11 \\
\hline & Change & & 0.27 & 0.25 & 0.511 & -0.29 & 0.86 \\
\hline \multirow[t]{3}{*}{ Leadership } & Pre & 2 & 2.07 & 2.07 & 0.303 & 1.86 & 2.29 \\
\hline & Post & & 4.21 & 4.21 & 0.303 & 4.00 & 4.43 \\
\hline & Change & & 2.14 & 2.14 & 0.000 & 2.14 & 2.14 \\
\hline \multirow{3}{*}{$\begin{array}{l}\text { Interprofessional Team } \\
\text { Collaboration }\end{array}$} & Pre & 4 & 3.81 & 4.00 & 0.389 & 3.22 & 4.00 \\
\hline & Post & & 4.19 & 4.00 & 0.389 & 4.00 & 4.78 \\
\hline & Change & & 0.39 & 0.39 & 0.449 & 0.00 & 0.78 \\
\hline \multirow[t]{3}{*}{ Patient-Centered Care } & Pre & 4 & 3.57 & 3.57 & 0.495 & 3.14 & 4.00 \\
\hline & Post & & 4.25 & 4.00 & 0.500 & 4.00 & 5.00 \\
\hline & Change & & 0.68 & 0.86 & 0.457 & 0.00 & 1.00 \\
\hline \multirow[t]{3}{*}{ Shared Decision Making } & Pre & 4 & 3.75 & 3.93 & 0.410 & 3.14 & 4.00 \\
\hline & Post & & 4.25 & 4.00 & 0.500 & 4.00 & 5.00 \\
\hline & Change & & 0.50 & 0.43 & 0.589 & 0.00 & 1.14 \\
\hline \multirow[t]{3}{*}{ Sustained Relationships } & Pre & 2 & 3.67 & 3.67 & 0.707 & 3.17 & 4.17 \\
\hline & Post & & 4.50 & 4.50 & 0.707 & 4.00 & 5.00 \\
\hline & Change & & 0.83 & 0.83 & 0.000 & 0.83 & 0.83 \\
\hline \multirow{3}{*}{$\begin{array}{l}\text { Quality Improvement and } \\
\text { Population Management }\end{array}$} & Pre & 1 & 3.20 & 3.20 & & & \\
\hline & Post & & 3.80 & 3.80 & & & \\
\hline & Change & & 0.60 & 0.60 & & & \\
\hline
\end{tabular}




\section{Journal Of Nursing Practice}

http://thejnp.org

ISSN: 2614-3488 (print); 2614-3496 (online)

Vol.3 No.2. April 2020. Page.253-267

Table 3. Results of Paired t-Tests for Changes in Competency Tool Domain Scores

\begin{tabular}{llll}
\hline Competency Tool Domain & $\mathrm{t}$ & $\mathrm{df}$ & $\mathrm{p}$ \\
\hline Clinical Competency in Planning and Managing Care & 1.052 & 3 & 0.370 \\
\hline Interprofessional Team Collaboration & 1.732 & 3 & 0.182 \\
\hline Patient-Centered Care & 2.967 & 3 & 0.059 \\
\hline Shared Decision Making & 1.698 & 3 & 0.188 \\
\hline
\end{tabular}

Figures: Bar Charts - Pre and Post Competency Tool Data Analysis

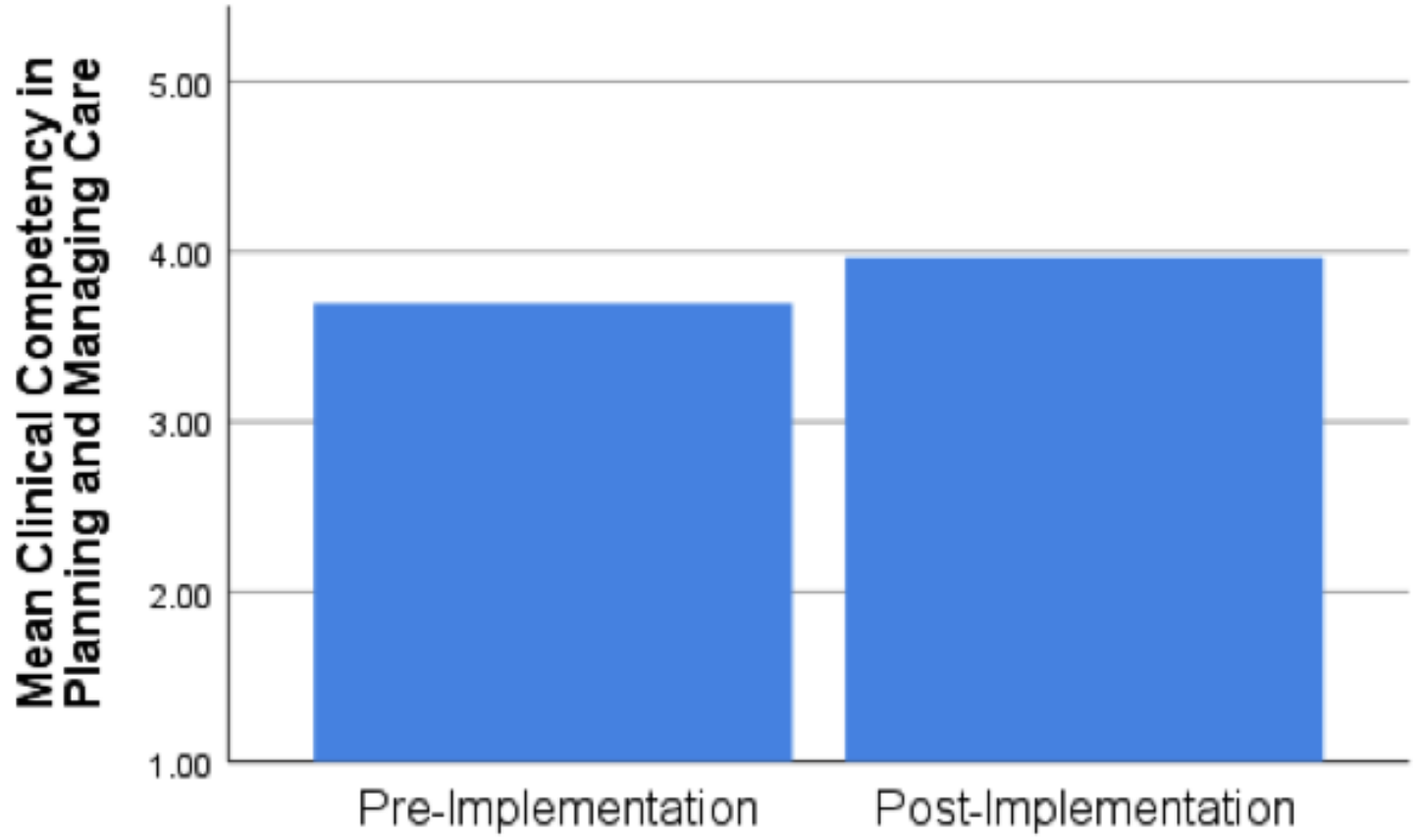

Clinical Competency in Plannıng and Managıng C'are Domain Scores, Pre- and PostImplementation

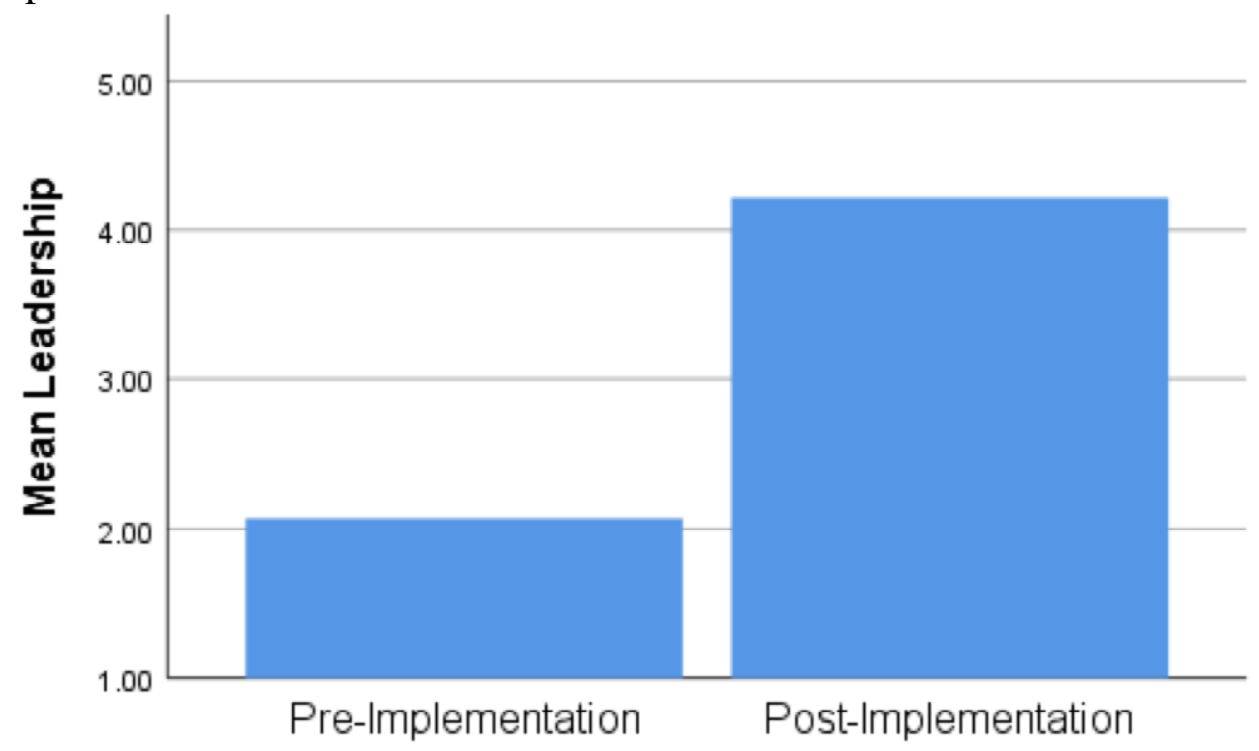




\section{Journal Of Nursing Practice}

http://thejnp.org

ISSN: 2614-3488 (print); 2614-3496 (online)

Vol.3 No.2. April 2020. Page.253-267

Figure 2. Bar Chart of Mean Leadership Domain Scores, Pre- and Post-Implementation

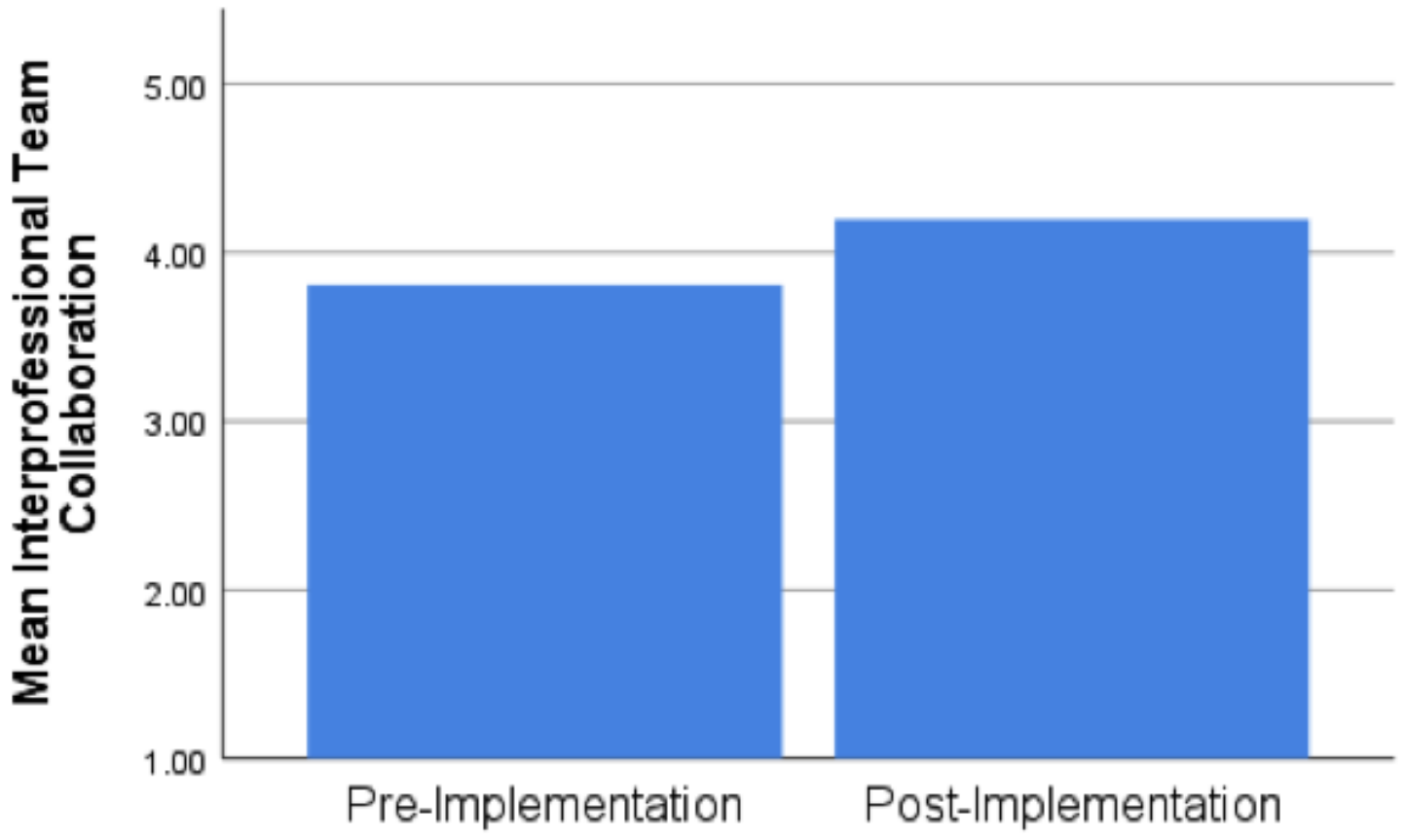

Figure 3. Bar Chart of Mean Interprofessional Team Collaboration Domain Scores, Preand Post-Implementation

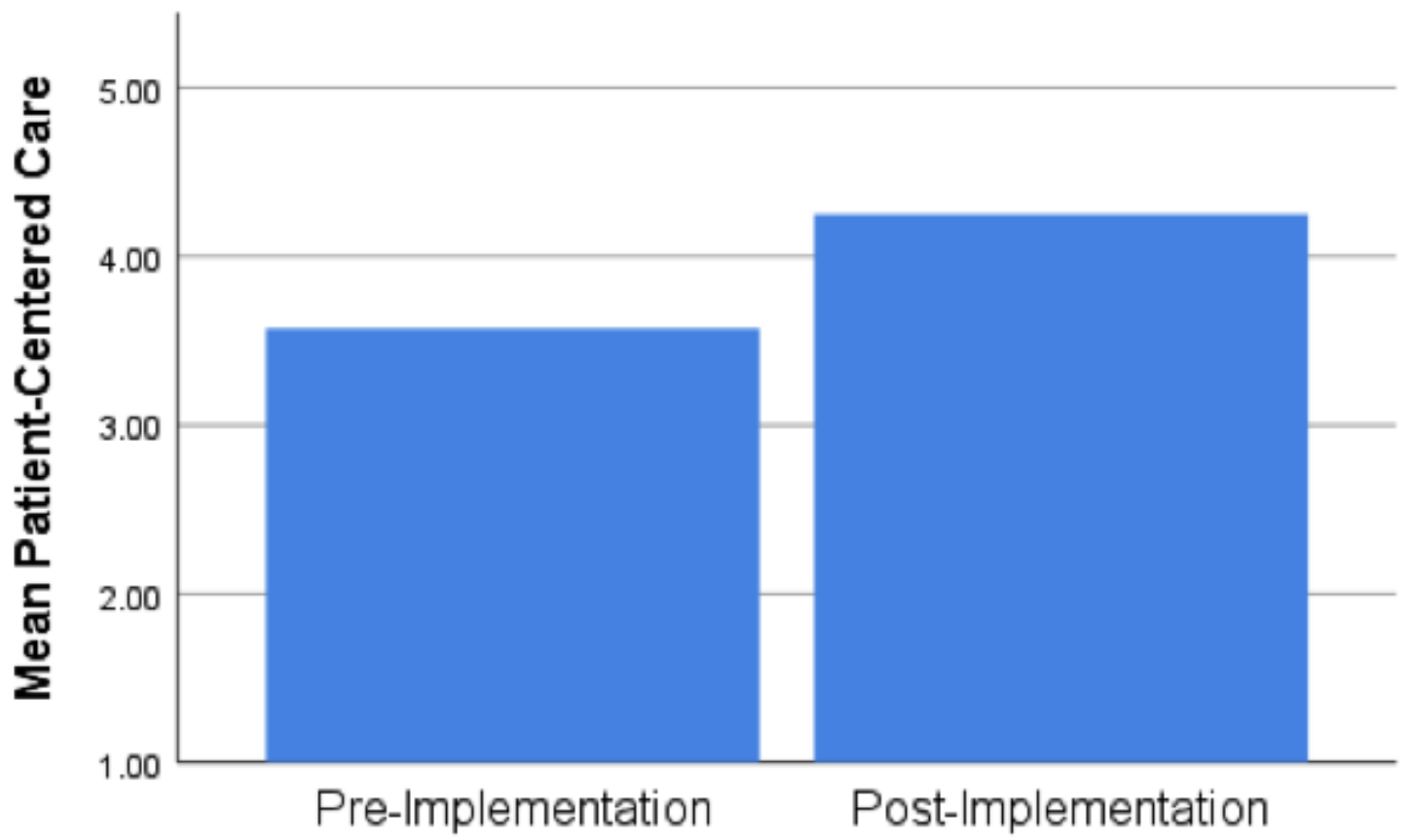

Figure 4. Bar Chart of Mean Patient-Centered Care Domain Scores, Pre- and PostImplementation 


\section{Journal Of Nursing Practice}

http://thejnp.org

ISSN: 2614-3488 (print); 2614-3496 (online)

Vol.3 No.2. April 2020. Page.253-267

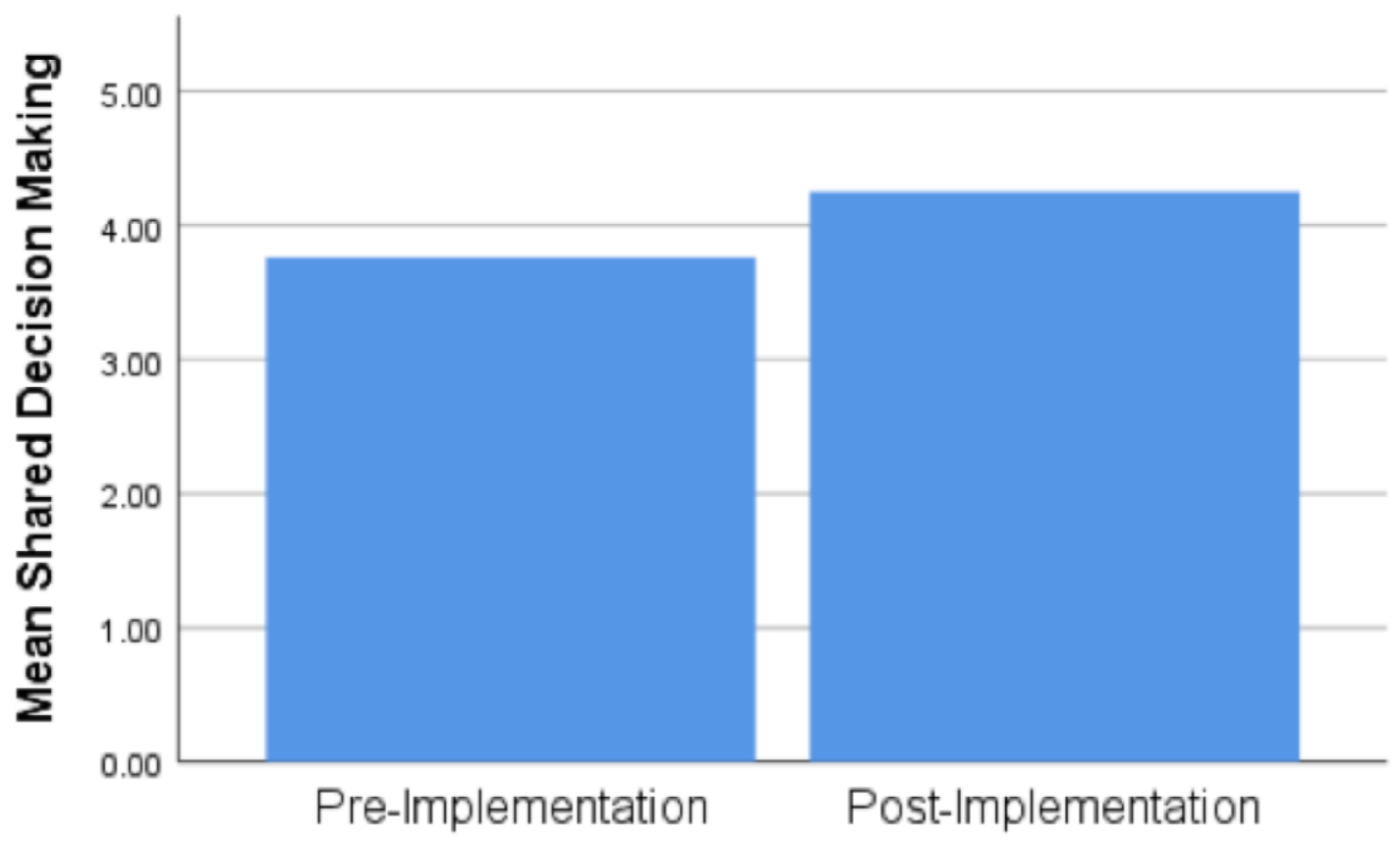

Figure 5. Bar Chart of Mean Shared Decision Making Domain Scores, Pre- and PostImplementation

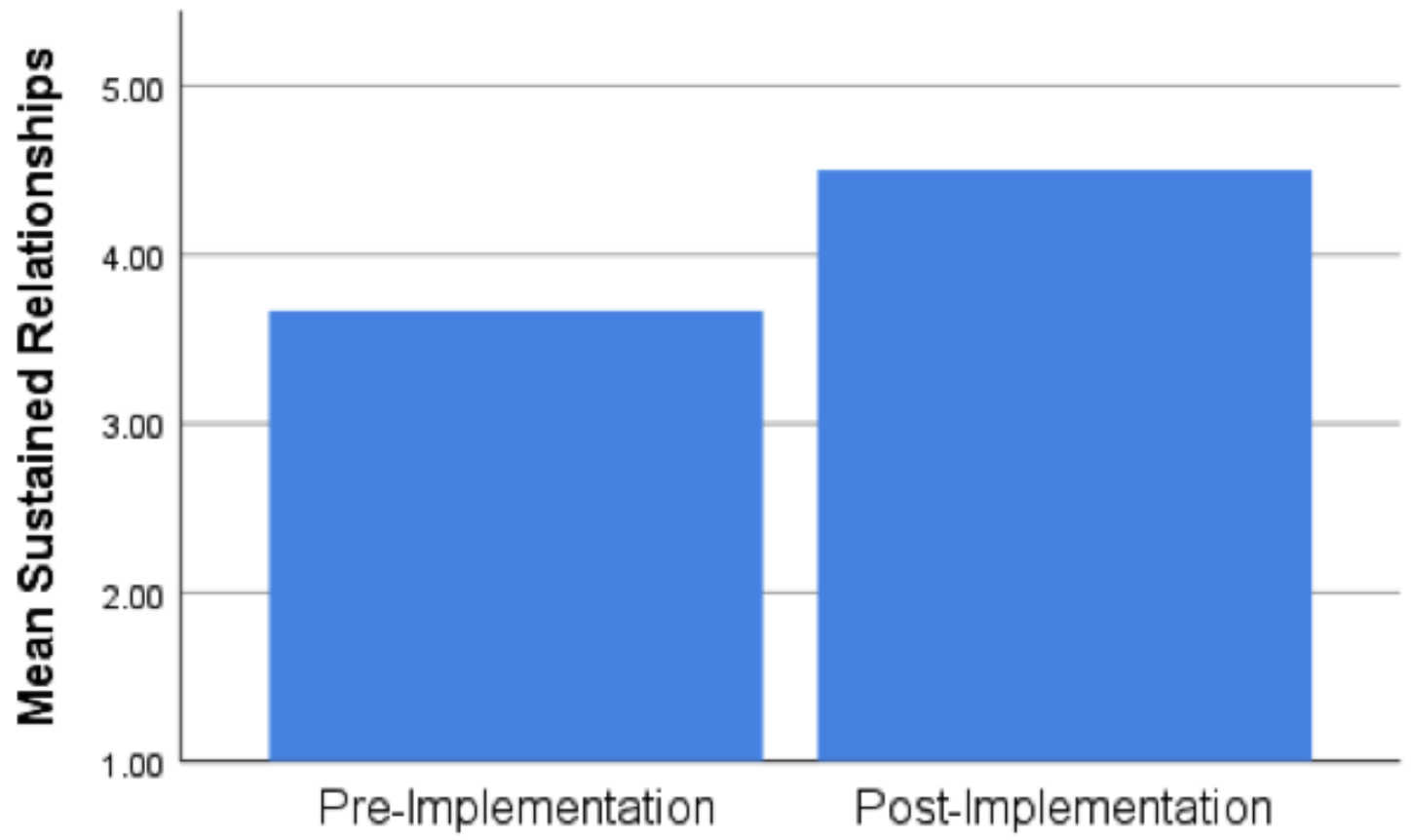

Figure 6. Bar Chart of Mean Sustained Relationships Domain Scores, Pre- and PostImplementation 
Journal Of Nursing Practice

http://thejnp.org

ISSN: 2614-3488 (print); 2614-3496 (online)

Vol.3 No.2. April 2020. Page.253-267

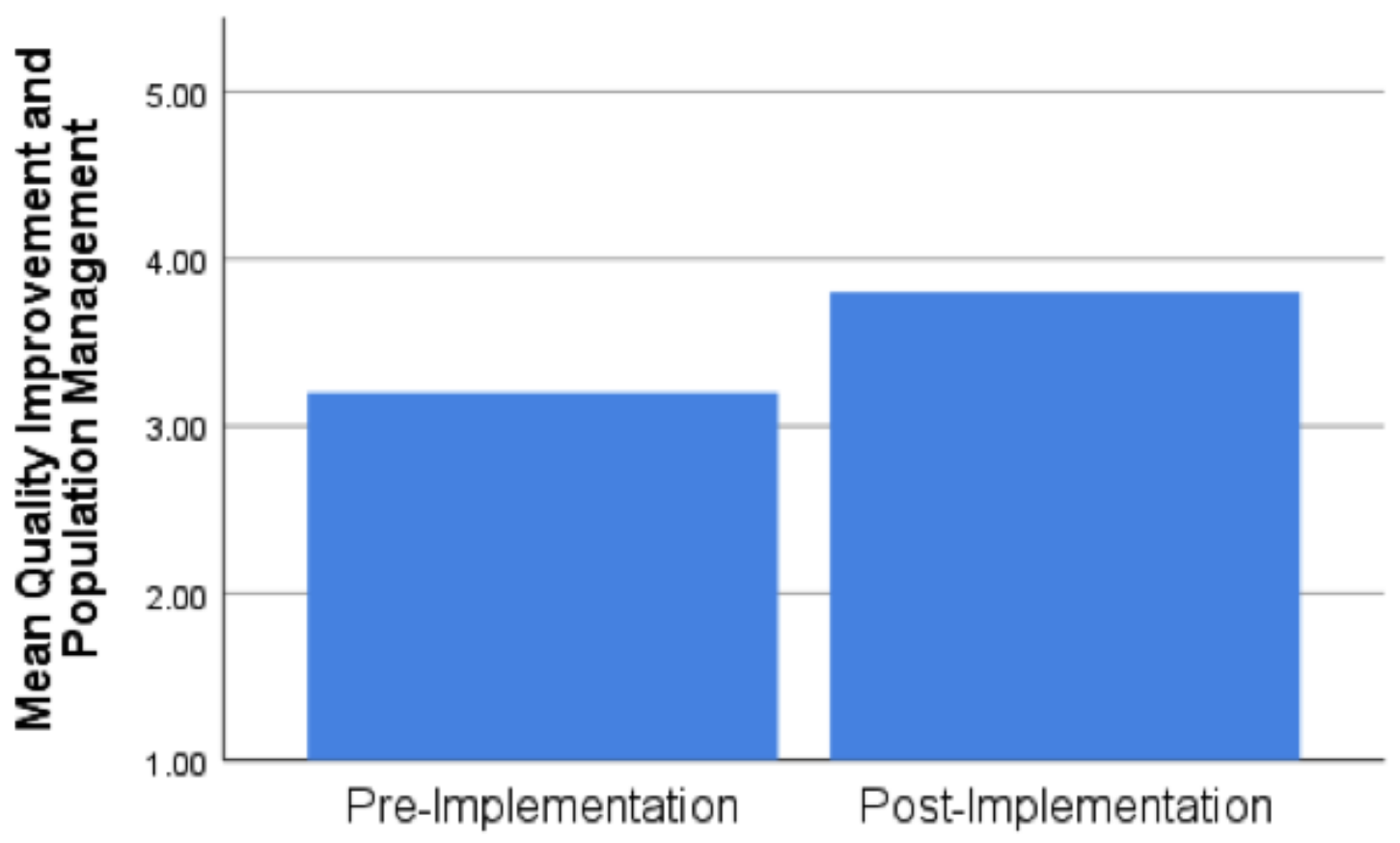

Figure 7. Bar Chart of Mean Quality Improvement and Population Management Domain Scores, Pre- and Post-Implementation 